\title{
SPANXA suppresses EMT by inhibiting c-JUN/SNAI2 signaling in lung adenocarcinoma
}

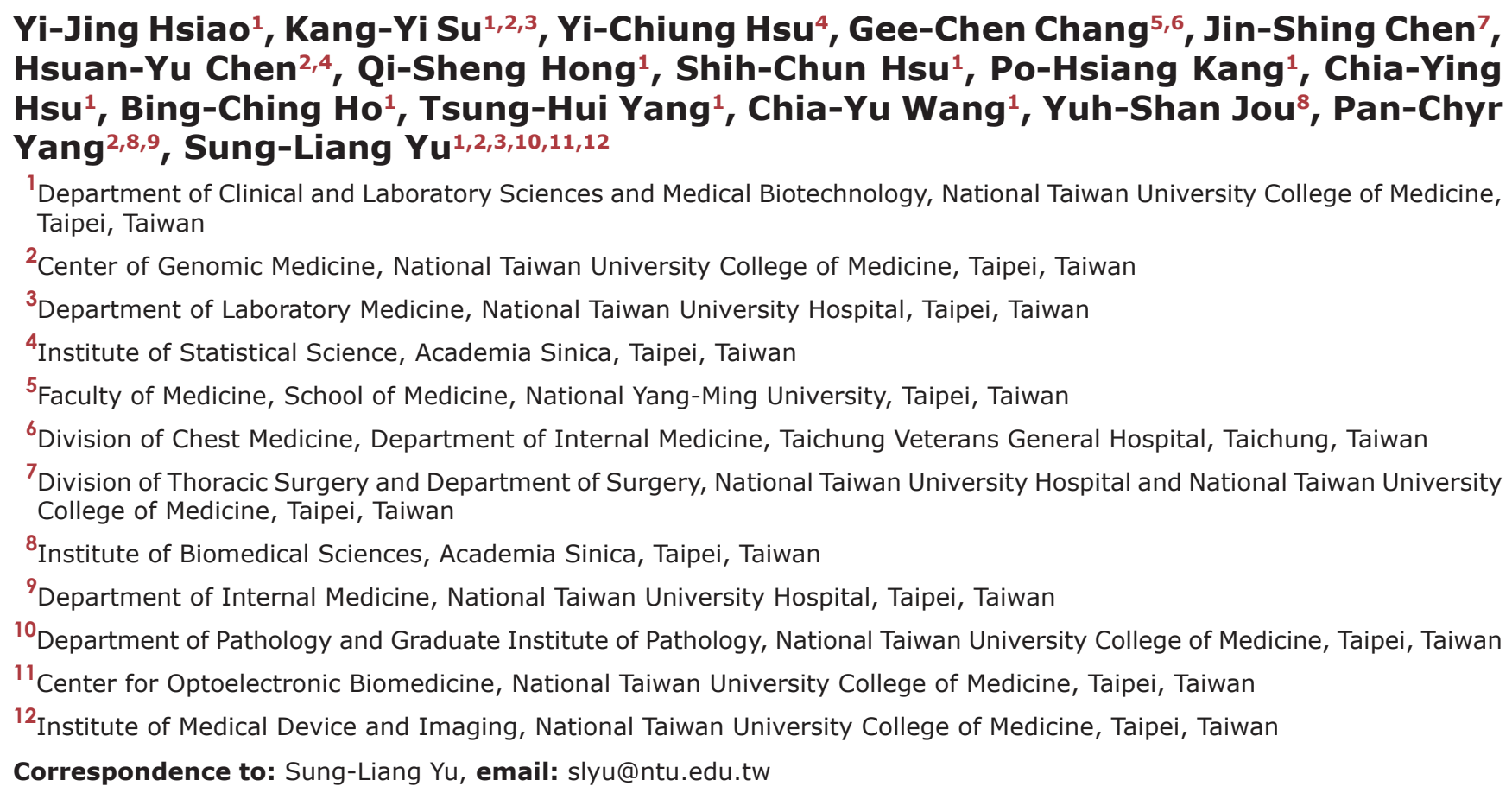
Keywords: SPANX family, AP-1, SLUG, E-cadherin, metastasis Received: January 07, $2016 \quad$ Accepted: June 01, $2016 \quad$ Published: June 15, 2016

\section{ABSTRACT}

SPANXA (Sperm Protein Associated with the Nucleus on the X-chromosome, family members A1/A2) acts as a cancer-testis antigen expressed in normal testes, but dysregulated in various tumors. We found that SPANXA is highly expressed in low-invasive CL1-0 cells compared with isogenous high-invasive CL1-5 cells. SPANXA was preferably expressed in tumor tissues and associated with the prolonged survival of lung adenocarcinomas. SPANXA suppressed the invasion and metastasis of lung cancer cells in vitro and in vivo. By the expression microarray and pathway analysis, we found that the SPANXA-altered genes were enriched in the epithelialmesenchymal transition (EMT) pathway. SPANXA reduced SNAI2 expression resulted in up-regulating E-cadherin. C-JUN acts as the positive-regulator of EMT. Silencing SPANXA increased C-JUN mRNA expression and blockage of c-JUN led to SNAI2 down-regulation. Our results clearly characterized SPANXA as an EMT inhibitor by suppressing $c-J U N-S N A I 2$ axis in lung adenocarcinoma.

\section{INTRODUCTION}

Lung cancer is the leading cause of cancer death worldwide, and adenocarcinoma is the major subtype, accounting for $50 \%$ of the incidence of non-small cell lung cancer (NSCLC) [1, 2]. Metastasis is one major hurdle in cancer treatment [3]. During metastasis, cancer cells acquire their invasive capacity by undergoing epithelialmesenchymal transition (EMT), which enables cells to become motile, and thus, invade adjacent vessels and tissues. The epithelial cells lose cell-cell junctions by down-regulating E-cadherin (CDH1) while acquiring a mesenchymal phenotype, which is characterized by upregulated mesenchymal proteins, including vimentin 
(VIM), N-cadherin (CDH2) and fibronectin (FN1). This process is triggered by various signaling pathways controlled by a set of transcription factors, including SNAI1/2, HEY1, TWIST and ZEB1/2 [4-6]. In lung cancer, SNAI2/SLUG is known as a critical EMT inducer that suppresses $\mathrm{CDH} 1$ expression and metastasis $[7,8]$. In our previous studies, we established a series of lung cancer cell lines CL1-0, CL1-1, CL1-2 and CL1-5, with various malignancies and invasiveness [9]. Using this cell line model and microarray analysis, we identified several invasion-related genes, and characterized their roles in metastasis $[7,10-12]$. It is widely now accepted that drug resistance and metastasis of cancer is largely caused by the dysregulation of certain genes [13]. Therefore, we reanalyzed the microarray data of our cell line model and identified SPANXA belonging to cancer-testis antigens (CTAs). The SPANX (Sperm Protein Associated with the Nucleus on the X-chromosome) family encodes highly similar proteins that are exclusively expressed in normal testes as well as in certain tumors [14-17]. In adult human testes, SPANXA/D (A1, A2, B, C, and D) proteins are localized on the nuclear envelope of condensing nonacrosomal regions during spermiogenesis, and expressed in late spermatids and spermatozoa $[18,19]$. SPANXA/D proteins have also been found in various tumors, such as melanoma, bladder carcinomas, myeloma, head and neck squamous cell carcinoma (HNSCC), as well as colorectal and prostate cancers [14, 20-23]. However, their roles in spermiogenesis and cancer progression are largely unknown. The nucleotide sequences of the SPANXA/D subfamily generally share similarities of $80 \%-90 \%[15,24]$. Moreover, the sequence of SPANXA1 is identical to that of SPANXA2, including introns. Both genes are located at the opposite orientation within the contiguous sequence of Xq27 [17]. Therefore, SPANXA1 and SPANXA2 encode the same mRNA and protein, and the biological functions remain to explore.

In this study we analyzed the clinical relevance of SPANXA in lung cancer patients by using the published microarray dataset and Taiwan lung cancer cohort with real-time quantitative reverse transcriptase polymerase chain reaction (qRT-PCR). The effect of SPANXA on metastasis was characterized by in vitro and in vivo assays. We finally explore the underlying mechanism by which SPANXA regulates the downstream signaling through microarray analysis.

\section{RESULTS}

\section{SPANXA is upregulated in tumor tissues and associated with prolonged survival in lung adenocarcinoma patients}

In our previous studies, we analyzed a series of lung adenocarcinoma cell lines with varying degrees of invasiveness by expression microarrays to identify novel tumor suppressor genes or oncogenes $[7,9,10,12]$. As with the previous strategy, in a comparison of the expression profiles of low-invasive CL1-0 cells and highinvasive CL1-5 cells, we found a gene, SPANXA, which expression was 102 times lower in CL1-5 than in CL1-0 (Supplementary Figure S1). At first, we assessed whether the SPANXA expression is associated with survival of lung adenocarcinoma patients. To address this issue, we conducted a survival analysis using the publicly available microarray dataset, GSE19188. There was a significant correlation between SPANXA expression and overall survival in the dataset of 45 lung adenocarcinoma patients. Patients with low SPANXA expression had a worse overall survival compared with those with high SPANXA expression (log rank test, $p=0.03$, Supplementary Figure S2A). However, the detection specificity of SPANXA should be carefully considered cause of the high similarity among the SPANX family. Thus we designed the probes of real-time qRT-PCR to detect SPANXA and distinguish from other SPANX family, especially SPANXC that has only seven nucleotides different from SPANXA. First, both SPANXA-V5 and SPANXC-V5 expression plasmids were constructed and transfected into HEK293 cells (Supplementary Figure S3A). The customized SPANXA and SPANXC TaqMan probes as well as SYBR primers detected the corresponding SPANX genes with high specificity, respectively (Supplementary Figure S3B and $\mathrm{S} 3 \mathrm{C})$. Consistent with expression microarray, the differential expression of SPANXA in CL1-0 and CL1-5 was further confirmed by qRT-PCR with the TaqMan probe (Supplementary Figure S3D). Next we measured the expression of SPANXC in CL1-0 and CL1-5 cells and found that the SPANXC expression is much lower than SPANXA in both cell lines even if SPANXC is expressed (Supplementary Figure S4). These data indicated that SPANXC does not play an anti-metastatic role at least in CL1-0 and CL1-5 cells. Following experiments, we used SYBR primers to quantify SPANXA expression, except for additional notation.

Next, SPANXA expression in 97 paired adjacent normal and tumor tissues from the non-small cell lung cancer patients were measured by qRT-PCR with the TaqMan probe (Supplementary Table S1). The result showed that SPANXA was dominantly present in tumor tissues (McNemar test, $p=0.019$, Table 1) and the following subtype stratification found that SPANXA expression was upregulated in adenocarcinoma patients (Wilcoxon matched-pairs method, $p=0.028$, Supplementary Figure S2B).

\section{SPANXA suppresses cell motility and invasiveness in vitro}

The role of SPANXA in lung cancer progression has never been fully elucidated. The SPANXA-V5 expression plasmid was transfected into the low 


\begin{tabular}{|c|c|c|c|c|c|}
\hline & & \multicolumn{3}{|c|}{ Normal tissue } & \multirow[b]{2}{*}{$p$-value ${ }^{\#}$} \\
\hline & & Presence* & Absence* & Total & \\
\hline \multirow{3}{*}{ Tumor tissue } & Presence & 5 & 15 & 20 & \multirow{3}{*}{0.019} \\
\hline & Absence & 4 & 73 & 77 & \\
\hline & Total & 9 & 88 & 97 & \\
\hline
\end{tabular}

*CT of SPANXA greater than 40 cycles was defined as Absence, otherwise as Presence.

\#McNemar's test was used for the statistical analyses.

SPANXA-expressing CL1-5 and EKVX cells to establish pooled stably SPANXA-expressing cells (Figure 1A and Supplementary Figure S5A). Transwell migration and invasion assays indicated that SPANXA inhibits both migration and invasion abilities in stably SPANXAexpressing cells, CL1-5 and EKVX cells (Figure 1B and $1 \mathrm{C}$ ) as well as in transient SPANXA-expressing CL1-5 cells (Supplementary Figure S5B) and single clones (Supplementary Figure S5C and S5D). To dissect the underlying cause of SPANXA-mediated invasion suppression, the cell growth evaluation was measured in SPANXA manipulated cell lines. The MTT assay indicated SPANXA does not influence cell proliferation (Supplementary Figure S5E). The single-cell tracking assay revealed that SPANXA suppresses cancer cell motility (Figure 1D). These data clarified that SPANXA inhibits cell migration and invasion.

\section{Downregulated SPANXA promotes cell migration and invasiveness}

To evaluate the knockdown efficacy, five shSPANXA lentiviruses which targeted different sites of SPANXA were used to infect the SPANXA-expressing HEK293 cells. Only shSPANXA-4 (sh4) reduced the SPANXA expression efficiently (Supplementary Figure S6A). Silencing SPANXA enhanced cell migration and invasion in enforced SPANXA-expressing CL1-5 cells (Figure 2A and Supplementary Figure S6B), and in CL1-0 and H1437, which both were highly endogenous SPANXA cells lines (Figure 2B, 2C and Supplementary Figure $\mathrm{S} 5 \mathrm{~A}$ ). In addition to cell migration and invasion, we also investigated whether SPANXA influences tumorigenesis in vitro. In anchorage-independent assay, SPANXAexpressing cells formed fewer colonies compared with the mock (Supplementary Figure S6C). These data demonstrated that downregulated SPANXA increases cell migration and invasion in lung adenocarcinoma cells.

\section{SPANXA inhibits metastasis in vivo}

To evaluate the role of SPANXA in vivo, we injected the stably SPANXA-expressing or mock control cells into the lateral tail vein of NOD-SCID mice. The mice were sacrificed after five weeks, and the lungs were examined for metastasis formation. Compared with mock mice, the nodules decreased significantly in SPANXA mice (Figure 3A and 3B). The representative lungs were stained with hematoxylin and eosin (H\&E) for histological analysis (Supplementary Figure S7). These results revealed that SPANXA plays a role in metastasis suppression in vivo.

\section{SPANXA is mainly involved in EMT pathway}

To dissect the underlying mechanism through which SPANXA suppresses metastasis, we performed oligonucleotide expression microarrays to profile differentially expressed genes in stably SPANXAexpressing cells. There were 1024 genes with a 2-fold change $(p<0.05)$ applying to MetaCore enrichment pathway analysis software, and the top 10 most affected pathways were identified (Supplementary Table S2). Surprisingly, the altered genes were largely enriched to EMT pathway: four pathways belonged to EMT pathways and five were EMT-related pathways. Following verification, the cell morphology of stably SPANXA-expressing cells changed markedly into the epithelial type from the original CL1-5 mesenchymallike type (Figure 4A). The F-actin remodeling occurred in the SPANXA-expressing cells, which evidently had less filapodia compared with the mock control (Figure 4B). The anti-V5 immunoflorescence staining results indicated that SPANXA localizes mostly in the nucleus (Figure 4B). When SPANXA was overexpressed in CL1-5 cells, the epithelial marker, E-cadherin was upregulated, whereas other mesenchymal proteins were downregulated (Figure 4C). Otherwise, knockdown of SPANXA expression reduced the protein and mRNA expression of E-cadherin, and increased mesenchymal markers N-cadherin, $\beta$-catenin and Vimentin in CL10 cells (Supplementary Figure S8A and S8B). Overall, these data clearly demonstrated that SPANXA negatively regulates EMT in lung cancer cells.

\section{SPANXA inhibits cell invasion by reducing SNAI2/SLUG expression}

Through microarray analysis, we identified several genes involved in the EMT-related pathways; one 
was SNAI2, which was downregulated to 0.22 -fold in SPANXA-expressing cells (Supplementary Table S3). The transcriptional factor SNAI2 was a critical EMT inducer that suppresses its target, E-cadherin. Both mRNA and protein expressions of $S N A I 2$ were obviously reduced in the stably SPANXA-expressing cells compared with the mock control cells (Figure 5A). As expected, E-cadherin was also downregulated detected by microarray and qRT-PCR assays (Supplementary Table S3). By contrast, silencing SPANXA in CL1-0 cells enhanced SNAI2 expression and reduced E-cadherin expression (Figure 5B and Supplementary Figure S8B). Moreover, SPANXA knockdown in stably SPANXA-expressing cells rescued SNAI2 downregulation (Figure 5C). To clarify the role of SNAI2 in SPANXA-mediated invasion suppression, we ectopically expressed SNAI2 in stably SPANXAexpressing cells and assessed cell invasion. The invasive ability suppressed by SPANXA was partly regained by restoring SNAI2 (Figure 5D). By contrast, when we used siRNA to suppress SNAI2 expression, which was upregulated in stably SPANXA-silencing CL1-0 cells, the invasive ability was inhibited (Figure 5E). These results revealed that SPANXA inhibits cell invasion by transcriptionally reducing SNAI2 expression.

\section{SPANXA suppresses c-JUN expression leading to SNAI2 downregulation}

Previous studies reported that the $\beta$-catenin/ TCF is responsible for transcription of SNAI2 $[25,26]$. Although SPANXA reduced $\beta$-catenin expression, silencing $\beta$-catenin did not significantly decrease the SNAI2 expression in SPANXA-silencing CL1-0 cells (Supplementary Figure S9). To identify which transcriptional factor responsible for $S N A I 2$ suppression, we analyzed 1024 SPANXA-altered genes from the microarray to build the SPANXA-regulating network by transcriptional regulation analysis in MetaCore. The SNAI2-involved network was sorted out. We found c-JUN to be the key mediator in this network, and these genes in the network are enriched in cell motility $(p=1.20 \mathrm{e}-22)$ (Supplementary Table S4). Generally,

A
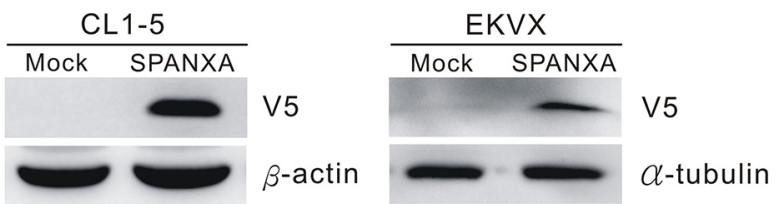

B
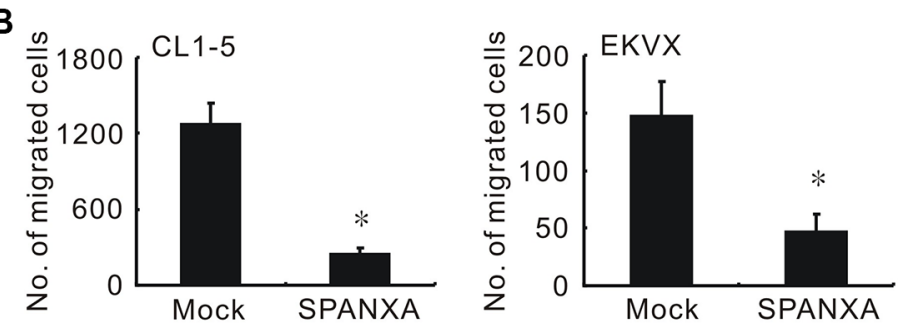

C
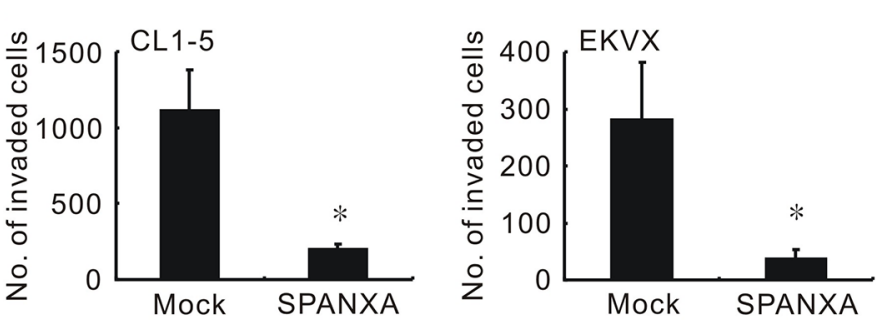

D

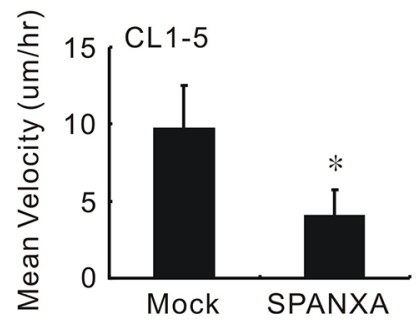

Figure 1: SPANXA suppresses cell migration and invasion. (A) Establishment of SPANXA-expressing cells in highly invasive cells, CL1-5, and in low SPANXA-expressing cells, EKVX. The SPANXA expression was detected by anti-V5. (B) (C) Cell migration and invasion abilities of mock or SPANXA-expressing cells measured by Transwell assay. (D) Cell motility rate of SPANXA-expressing cells and mock control were measured by single cell tracking. All experiments were performed in triplicates. ${ }^{*} P<0.05(\mathrm{mean} \pm \mathrm{SD}, n=3)$. 
c-JUN dimerized with c-FOS to form AP-1. We first assessed c-JUN and c-FOS expressions in the stably cell lines. The data showed that SPANXA regulates c-JUN expression but not the c-FOS in both SPANXAexpressing and SPANXA-silencing cells (Supplementary Figure S10A and S10B). Additionally, the c-JUN mRNA is up-regulated in SPANXA-expressing cells and downregulated in SPANXA-silencing cells (Supplementary Figure S10C). The AP-1 reporter assay showed that SPANXA significantly decreases the AP-1 activity (Supplementary Figure S10D). These data suggested that SPANXA attenuates AP-1 activity mainly through c-JUN suppression. We delivered a different ratio of c-JUN and c-FOS expression plasmids into stably SPANXA-expressing CL1-5 cells. Re-expressed AP-1 increased $S N A I 2$ expression and reduced E-cadherin expression (Figure 6A and Supplementary Figure S11). Moreover, silencing c-JUN repressed SNAI2 upregulation

A

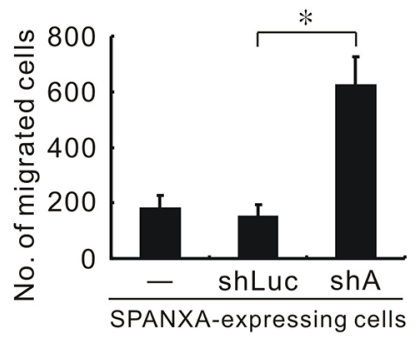

B

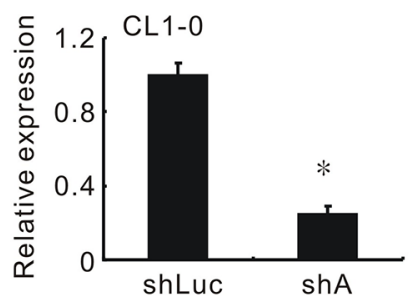

C
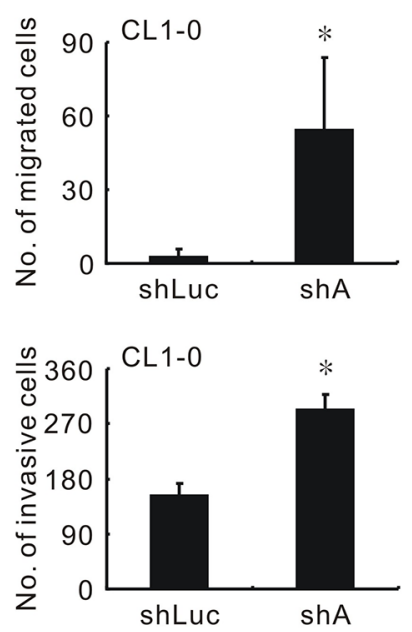

in SPANXA-silencing CL1-0 cells (Figure 6B). Next we identified the AP-1 binding motif (TGACTCA) in the promoter of human SNAI2 (NCBI accession No. NT_008183.20). The chromatin immunoprecipitation result showed that the region of AP-1 binding site is occupancy of c-JUN (Supplementary Figure S12). These results indicated that SPANXA inhibited SNAI2 expression via suppressing c-JUN at transcriptional level.

We evaluate the impact of SPANXA on the AP-1 transcriptional landscape by expression microarray and pathway analysis. There are 397 genes which are significantly down-regulated with 2-fold change in SPANXA-expressing cells; 583 genes are considered as AP-1/c-JUN regulating targets annotated by MetaCore software (GeneGo, St. Joseph, MI). The intersection of these AP-1/c-JUN targets and the SPANXA down-regulated genes are 31 genes. The SPANXA down-regulated genes are significantly enriched in
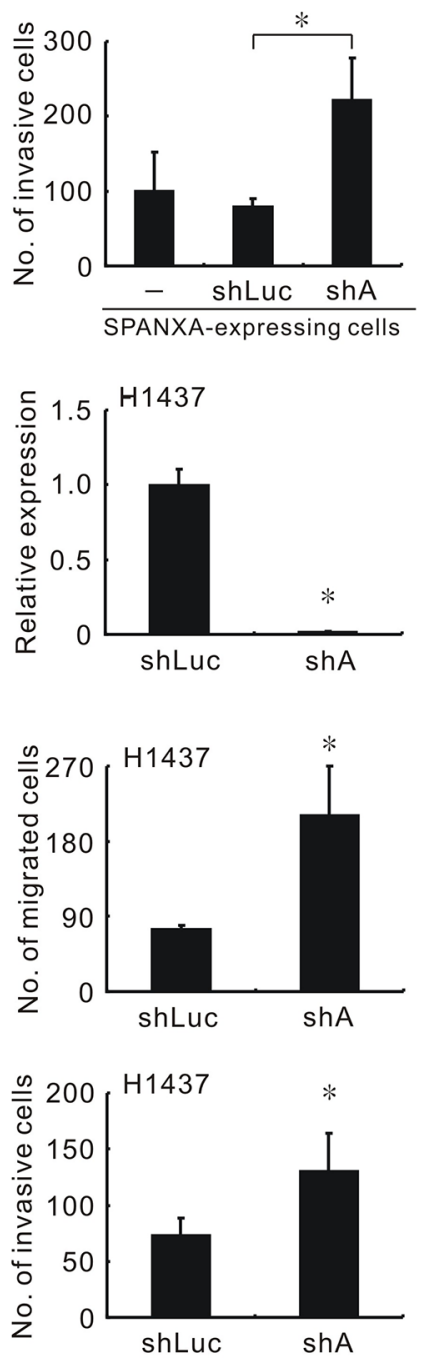

Figure 2: Knockdown of SPANXA increases cell migration and invasion in vitro. (A) Cell migration and invasion abilities of silencing SPANXA in stably SPANXA-expressing CL1-5 cells. (B) SPANXA expression in SPANXA-silencing CL1-0 and H1437 cells assessed by qRT-PCR. (C) Cell migration and invasion abilities of SPANXA-silencing CL1-0 and H1437 cells. All experiments were performed in triplicates. ${ }^{*} P<0.05($ mean $\pm \mathrm{SD}, n=3)$. 
AP-1/c-JUN regulating targets (Chi-square test, $p=1.50$ $\times 10^{-09}$, total gene number $=21,123$ ) (Supplementary Table S5). Thirty-one AP-1/c-Jun targets are downregulated in stably SPANXA-expressing cells listed (Supplementary Table S3). This data indicated that AP-1 alteration is a major function of SPANXA.

Figure 6C showed the signaling network of SPANXA-mediated tumor suppression in which SPANXA downregulates the c-JUN expression and a set of AP-1 targets associated with EMT, angiogenesis, chemoattraction and metastasis. The expression of EMTrelated genes was confirmed by qRT-PCR (Supplementary Table S3). These results revealed that SPANXA suppresses SNAI2 and reverses EMT through c-JUN suppression.

\section{DISCUSSION}

Cancer-testis antigens (CTAs) are a potential target for developing the anti-tumor vaccines by their testis/ tumor-biased expression pattern. Recently, emerged reports implied that certain CTAs are directly involved in tumorigenesis, metastasis and prognosis [27-29]. SPANX was first discovered as CTAs, which are widely distributed in tumors, such as in lung, liver, and colon cancers, as well as melanoma, but not in normal tissues, except for the testis $[14,16,30]$. The SPANX expression might be positively associated with metastasis in colorectal cancer and in metastatic melanoma $[17,31]$. A research indicated that SPANX was seemingly involved in cancer stem-like cells and malignant progression [32]. The members of SPANX family share a high similarity that restricts functional investigation for SPANXA. Herein, we characterize the biological role and clinical significance of SPANXA, and demonstrate SPANXA blocks EMT and leads to invasive suppression through suppressing AP1 signaling.
At first, we attempted to distinguish SPANXA from its family members. Beyond the limitation of a high degree of similarity between SPANXA and SPANXC, we tested the commercially available antibodies for SPANXA. However, they failed to detect the ectopic expression of SPANXA-V5. Salemi et al. underscored this difficulty in differentiating SPANX subfamilies by antibodies because of the high homology among SPANX family members [23]. Hence, we designed the SPANXA-specific qRTPCR probe/primer sets. We found that SPANXA was associated with prolonged overall survival of the patients with lung adenocarcinoma, and it was predominantly presented in lung tumor parts. This unique tumor/normal expression pattern of SPANXA is quite different from other tumor suppressors which are often down-regulated in tumors. The reason for SPANXA dysregulated in lung adenocarcinoma is still unclear. But our data clearly indicated that SPANXA is a metastasis suppressor in lung adenocarcinoma. To our best knowledge, this is the first study to characterize the functional role of SPANXA in cancers.

SPANXA is an acidic protein and associates with other nuclear envelope proteins, but shows no significant similarity with known nuclear proteins or nucleic acidbinding proteins in the spermatid $[16,19]$. These studies implied that SPANXA may interact with nucleic acids or other proteins in performing its functions. Indeed, our findings demonstrated that ectopic SPANXA is largely located at nucleus and suppresses SNAI2 at transcriptional level. And c-JUN is responsible for SNAI2 expression and EMT suppression. c-JUN is a basic region-leucine zipper protein belonging to one member of the AP-1 family. Transcription factor AP-1 is a dimer of c-JUN and c-FOS families [33]. The induction of c-JUN expression and activity leads to tumor progression and development [34].
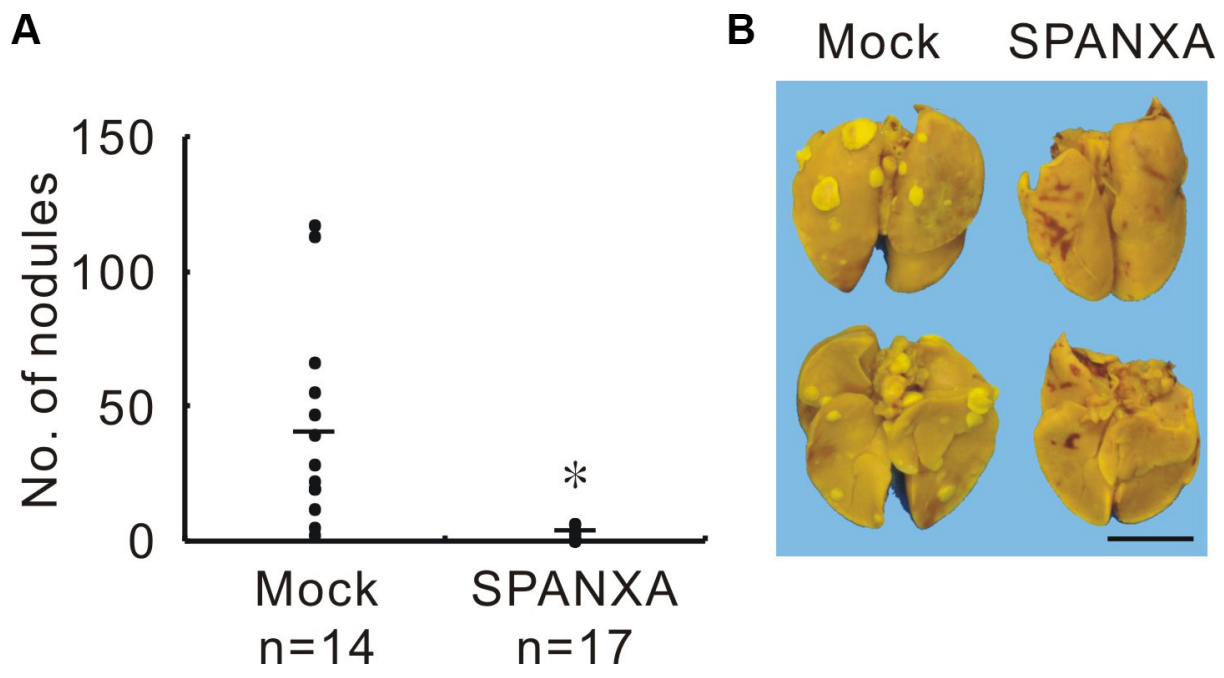

Figure 3: SPANXA attenuates metastasis in vivo. (A) In vivo tumor metastasis effect of SPANXA was analyzed by an experimental metastasis assay with stably SPANXA-expressing CL1-5 cells and mock control cells, which were intravenously injected into NOD-SCID mice. The metastatic tumor nodules were calculated. ${ }^{*} P<0.05$. (B) Image of lung surface nodules. Anterior lungs showed on the upper part and posterior lungs on the lower part. Scale bar, $0.5 \mu \mathrm{m}$. 
There are several AP-1 binding sites on SNAI2 promoter and SNAI2 is a direct transcriptional target of c-JUN in murine $[35,36]$. In breast cancer, SNAI2 was expressed in an AP-1-dependent manner [37]. Furthermore, c-JUN expression was found to parallel with SNAI2 expression, and correlated with a mesenchymal gene signature in a panel of melanoma cell lines and a patient cohort [38]. In this study we identified the AP-1 motif in the human promoter of SNIA2 occupancy of c-Jun and proved that the SPANXA-mediated EMT suppression is AP1dependent. These evidences suggest that c-JUN/SNAI2 signaling is a major pathway contributed to SPANXAmediated invasive suppression.

The SPANXA-inhibiting c-JUN targets are involved in metastasis, chemoattraction, angiogenesis and EMT (Figure 6C). Liu et al. reported that c-JUN induces the EMT process and directly activates a group of genes including TWIST2, TGM2, DUSP5 and ETS1 [39]. In agreement with this report, these four genes are down-regulated after ectopic SPANXA expression. In addition, several protumoral targets of AP-1/c-JUN were identified, implying that SPANXA suppresses tumorigenesis. For example, angiopoietin-2 (ANGPT2) is a proangiogenic protein facilitating neoangiogenesis [40]. ANGPT2 promoted metastasis of breast cancer through SNAI1 induction and E-cadherin inhibition [41]. High-mobility group protein B1 (HMGB1) is a chromosomal protein promoting tumor progression. HMGB1 is positively associated with the pathological grade and metastasis of liver cancer, and correlated with lung cancer patients' survival $[42,43]$. The SPANXAregulating network inhibits these protumoral genes that points out the importance of SPANXA. In fact, there are certain drawbacks in our study. Only one functional

A

Mock
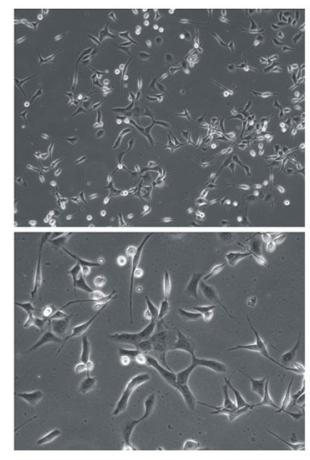

SPANXA

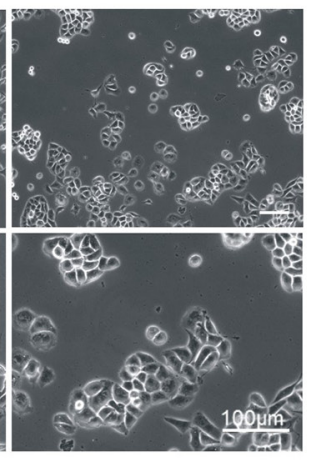

C

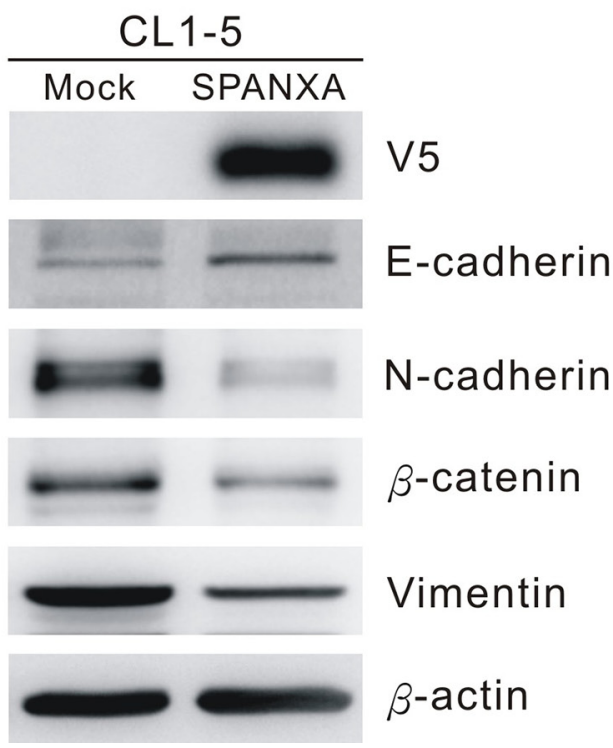

B
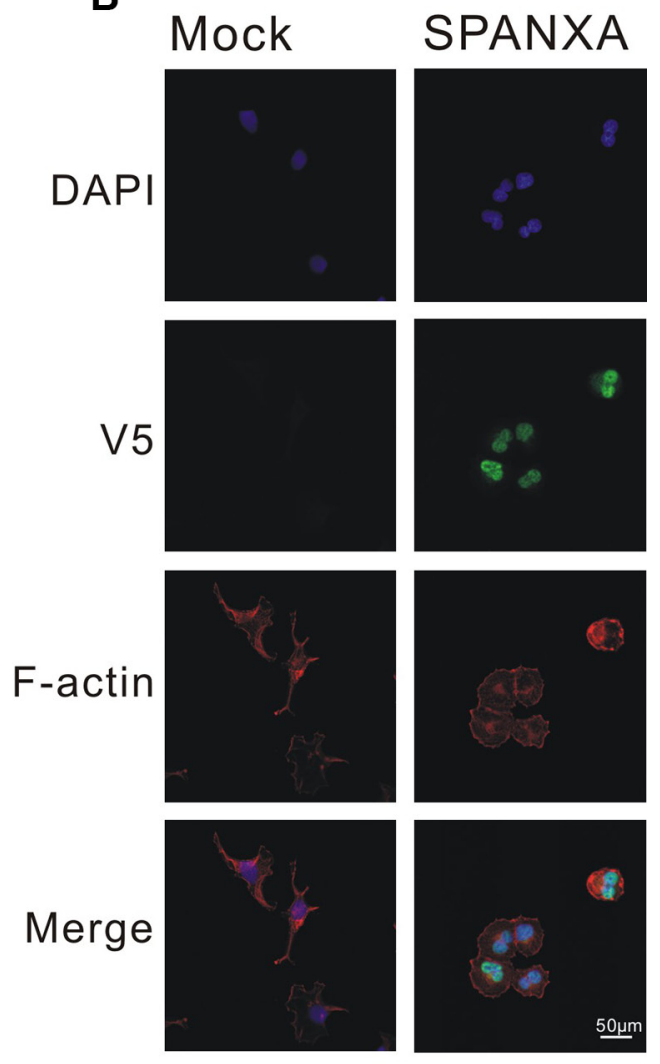

Figure 4: SPANXA inhibits EMT process. (A) Cell morphology of stably SPANXA-expressing CL1-5 cells and mock control cells. (B) Anti-V5 and anti-F-actin immunofluorescence staining of mock control and stably SPANXA-expressing CL1-5 cells. (C) Expression of EMT marker in stably SPANXA-expressing CL1-5 cells was assessed by immunoblot assays. All experiments were performed in duplicates. 
SPANXA shRNA may have an unavoidable off-target effect in the SPANXA-silencing experiments. Another concern was that we could not exclude the compensative effect attributed to other similar members. However,
SPANXA obviously suppressed EMT process, inhibited SNAI2 and c-JUN expressions in our model cell lines. The mechanism regarding how SPANXA suppresses c-JUN warrants further investigation. In conclusion,

\section{A}

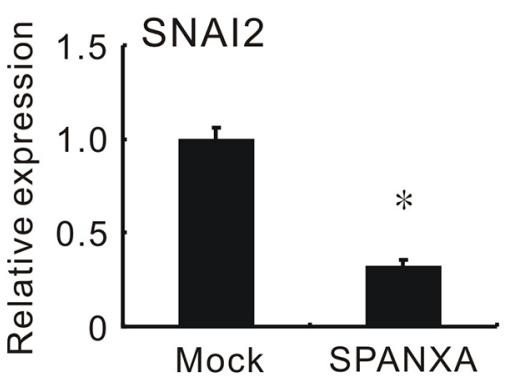

B

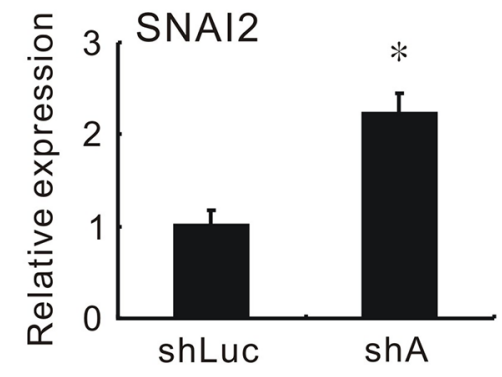

D

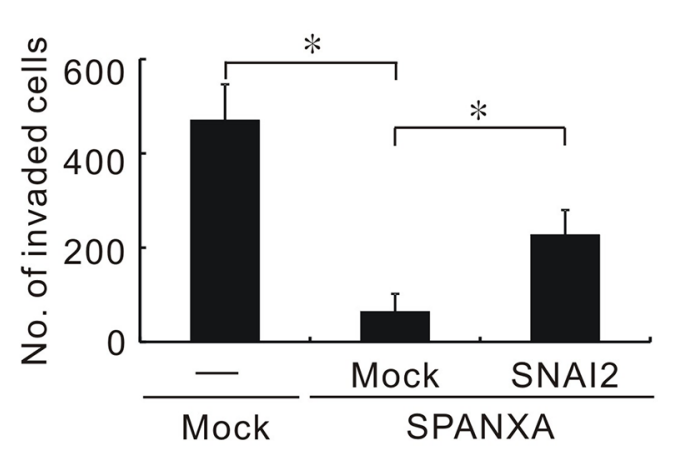

E

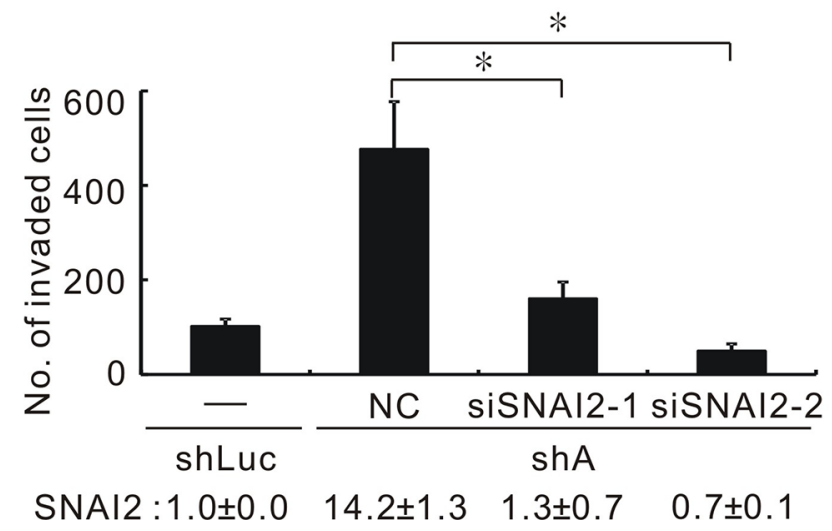

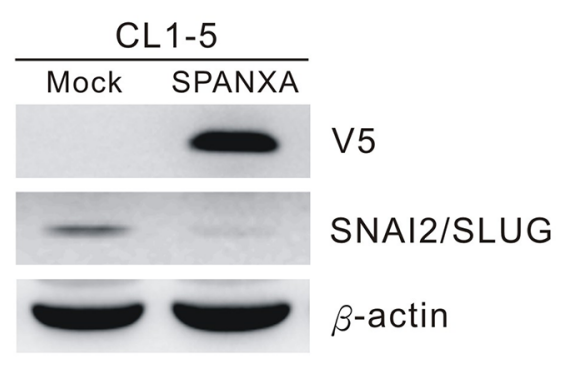

C

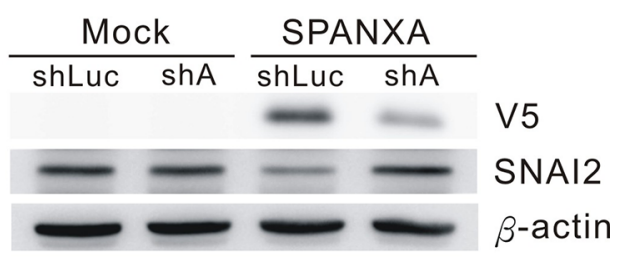

$\frac{\text { Mock }}{-} \frac{\text { SPANXA }}{\text { Mock SNAI2 }}$

E-cadherin

SNAI2

V5 $\beta$-actin

Figure 5: SPANXA represses cell invasion through deregulating SNAI2. (A) Protein and mRNA expressions of SNAI2 in stably SPANXA-expressing CL1-5 cells and mock control cells assessed by qRT-PCR and Western blot assays. ${ }^{*} P<0.05$ (mean \pm SD, $n=3$ ). (B) RNA expression of SNAI2 in SPANXA-silencing CL1-0 cells and shLuc control cells assayed by qRT-PCR. $* P<0.05$ (mean $\pm \mathrm{SD}, n=3$ ). (C) The stably SPANXA-expressing CL1-5 cells were infected with shSPANXA lentivirus and assayed for SNAI2 protein expression by Western blot assays. The experiment was performed in duplicates. (D) Cell invasion and expression of SNAI2 and E-cadherin of stably SPANXA-expressing CL1-5 cells was assessed in presence of SNAI2 overexpression. ${ }^{*} P<0.05$ (mean \pm SD, $n=3$ ) (E) Cell invasion of stably SPANXA-silencing CL1-0 cells was assessed under SNAI2 silencing. The lower row indicated SNAI2 RNA expression assayed qRT-PCR. $* P<0.05$ (mean $\pm \mathrm{SD}, n=3$ ). All assays were performed in two independent experiments. 
we provided evidence on and insight into the functional anti-metastasis roles of SPANXA, and demonstrated that SPANXA inhibits c-JUN/SNAI2 signaling resulting in
EMT reversal. These results suggest that SPANXA may be a potential target for therapeutic strategies targeting metastasis of lung cancer.
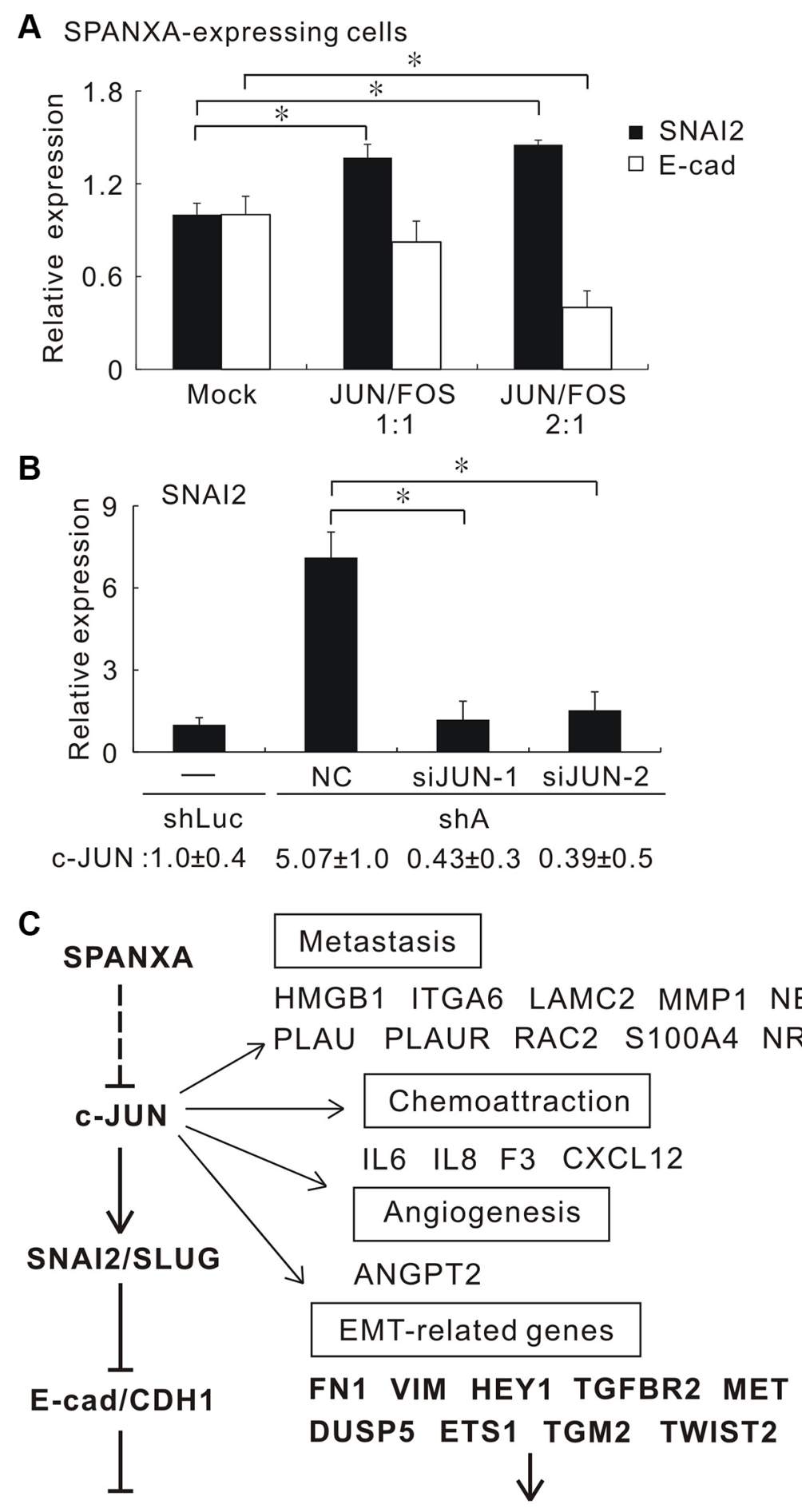

Cell Migration, Cell Invasion, Metastasis

Figure 6: SPANXA suppresses SNAI2 expression and EMT through repressing c-JUN. (A) The different ratios of c-JUN and c-FOS were ectopically introduced into stably SPANXA-expressing CL1-5 cells. The SNAI2 and E-cadherin expressions were measured by qRT-PCR. ${ }^{*} P<0.05$ (mean $\pm \mathrm{SD}, n=3$ ). (B) Knockdown of c-JUN in stably SPANXA-silencing CL1-0 cells reduced the $S N A I 2$ expression. The lower row indicated the $c-J U N$ expression. ${ }^{*} P<0.05$ (mean $\pm \mathrm{SD}, n=3$ ). (C) Model of SPANXA-regulating network. SPANXA inhibits the c-JUN/SNAI2/CDH1 signalling and EMT process. The dotted line indicates the unexplored part of SPANXA regulation. All assays were performed in two independent experiments. 


\section{MATERIALS AND METHODS}

\section{Clinical specimens}

Ninety-seven paired of lung cancer and adjacent normal lung tissues obtained from Taichung Veterans General Hospital and National Taiwan University Hospital were used to measure the SPANXA expression. All lung cancer patients were staged in accordance with the American Joint Committee on Cancer Staging (AJCC) and the histology was performed with World Health Organization standards. This study was approved by the institutional review board of Taichung Veterans General Hospital and National Taiwan University Hospital.

\section{Cell culture}

Human lung adenocarcinoma invasion model cell lines, CL1-0 and CL1-5, were established as described in our previous study [9]. Lung adenocarcinoma cell lines EKVX and H1437 were cultured in Dulbecco's Modified Eagle Medium (Life technologies, Carlsbad, CA) with $10 \%$ fetal bovine serum (Life technologies, Carlsbad, CA) and $1 \%$ penicillin and streptomycin $(100 \mathrm{mg} / \mathrm{mL})$. All cell lines were incubated at $37^{\circ} \mathrm{C}$ in a humidified $20 \% \mathrm{O}_{2} / 5 \%$ $\mathrm{CO}_{2}$ environment.

\section{Plasmid construction and transfection}

The SPANXA (GeneBank NM_013453) coding region was amplified and cloned into pEF6/V5-HisTOPO vectors (Life technologies, Carlsbad, CA). The sequence of SNAI2, c-JUN and c-FOS were cloned into the pcDNA3.1/V5-His-TOPO vectors. The cells were transfected with plasmids or siRNA by using Lipofectamine $^{\mathrm{TM}} 2000$ or RNAiMAX (Life technologies, Carlsbad, CA), in accordance with the manufacturer instructions. For transient transfection, the transfected cells were harvested $48 \mathrm{~h}$ later; for stably SPANXAexpressing transfectants, the cells were selected with 9 $\mathrm{mg} / \mathrm{mL}$ Blasticidin S; for stably SPANXA-repressing transfectants, cells were treated with $2.5 \mu \mathrm{g} / \mathrm{mL}$ puromycin (Life technologies, Carlsbad, CA).

\section{Lentiviral-based shRNA and siRNA}

The plasmids of shRNA for SPANXA were supplied by National RNAi Core Facility Platform (Academia Sinica, Taipei, Taiwan). They provided only 5 sequences to users. The clone ID of the fourth shRNA (sh4) for SPANXA was TRCN0000203935, and the target sequence was 5'-CGCTACAGGAGGAACTTTAAA-3'. The CL1-0 and $\mathrm{H} 1437$ cells were seeded onto $2 \times 10^{4}$ plates for $24 \mathrm{~h}$ before infection. Viral infection was performed according to the manufacturer protocol. SNAI2 and c-JUN siRNA were purchased from Invitrogen (Life technologies, Carlsbad, CA).

\section{Western blot analysis}

The quantitated cell lysates were separated on $8 \%-12 \%$ SDS polyacrylamide gels and transferred onto PVDF membranes. After blocking for $1 \mathrm{~h}$, the membranes were incubated sequentially with anti-V5 antibody (Life technologies, Carlsbad, CA), anti- $\beta$-actin antibody (Abcam, Cambridge, MA), anti-CDH1 antibody (BD Biosciences, San Diego, CA), anti-VIM antibody, anti- $\beta$-catenin ( Millipore, Temecula, CA), anti-CDH2 antibody (BD Bioscience, San Diego, CA), anti-SNAI2 antibody, anti-c-JUN antibody (Santa Cruz Biotechnology, Dallas, TX), and anti- $\beta$-tubulin antibody (Abcam, Cambridge, MA) in PBST. After washing for 3 times, the bound antibody was detected using the Enhanced Chemiluminescence System.

\section{In vitro migration and matrigel invasion assay}

The migration and invasion abilities were determined using a Transwell apparatus without or with Matrigel-coated membranes (BD Biosciences, Franklin Lakes, NJ). The migration and invasion assay procedure was detailed in a previous study [11]. The total number of attached cells was counted. All experiments were performed in triplicate. Single cell migration assay was performed as following description. Mock and stably SPANXA-expressing CL1 -5 cells were seeded 5000 cells/ per well in 6-well culture plate for overnight attachment. Wash wells with PBS twice and replace fresh medium. Set up the focus and sites for 25 position /per well, and take the picture every 1 hour in 24 hours. The high content screening software MetaMorph (Molecular Devices, Sunnyvale, CA) was used to analyze every single cell tracking and mean velocity.

\section{qRT-PCR}

The expression level of SPANXA was detected by qRT-PCR on ABI prism 7900 sequence detection system (Applied Biosystems, Branchburg, NJ), performed in accordance with the manufacturer instructions. For the SYBR Green methods, the SPANXA primers used were the following: forward primer SPANXA-F: 5'-AACGAGGCCAACGAGATGAT-3' and reverse primer SPANXA-R: 5'-CTAGTATGGTCGAGGACTCAGAT GTT-3' as well as the TATA box-binding protein (TBP) TBP-F: 5'-CACGAACCACGGCACTGATT-3' and TBP-R: 5'-TTTT CTTGCTGCCAGTCTGGAC-3'. TBP was used as the internal control. For TaqMan methods, the sequences of customized SPANXA and SPANXC detection probes were as follows: SPANXA forward primer: 5'-CGGGTCTGAGTCCCCAGTT-3', reverse primer: 5'- TC CCCTGTGATTCCAACGA-3', and the reporter sequence: 5'-CGGCATCATCTCGTTGGC-3'; SPANXC forward primer: 5'-CGGGTCTGAGTCCCCAGTT-3', reverse 
primer: 5'-TCCCCTGTGATTCCAACGA-3', and the reporter sequence: 5'-CGGCATCGTCTCATTCAC-3'. The TBP detection probe (Assay ID: Hs00427621_m1) was used as the internal control, which was supplied by the ABI company (Applied Biosystems, Branchburg, NJ). The relative expression level of interest, compared with that of TBP, was defined as $-\Delta \mathrm{CT}_{\text {Interest }}=-\left(\mathrm{CT}_{\text {Interest }}-\mathrm{CT}_{\mathrm{TBP}}\right)$, whereas the relative expression ratio of interest between different treatments was calculated as $2^{-\triangle \Delta C T}$. The primers of the EMT-related genes are listed in Supplementary Table S6.

\section{Microarray analysis}

For the microarray experiments, the total RNA of stably SPANXA-expressing CL1-5 cells and mock control was extracted and subjected to expression microarray analysis by using a Human Genome U133 Plus 2.0 GeneChip in accordance with the manufacturer instructions (Affymetrix, Santa Clara, CA). Experimental variations resulting from differences between microarrays were reduced by scaling the intensity values of the probes in each microarray by employing a quantile-normalization method. Raw data were normalized by GC-RMA and subjected to the unpaired Student $t$ test $(P<0.05)$ through GeneSpring GX 12.6 (Silicon Genetics, Redwood City, CA). The 1024 genes (2-fold change) that were expressed significantly between groups were subjected to pathway analysis by using MetaCore (version 6.16, GeneGo, St. Joseph, MI). These array data were uploaded onto GEO (GSE72323).

\section{Metastasis in SCID mice}

For in vivo metastasis assay, 6-week-old severe combined immunodeficiency (SCID) mice (supplied by the animal center at the College of Medicine, National Taiwan University, Taipei, Taiwan) were housed (6 mice per cage) and fed ad libitum with autoclaved food. All of the experiments were approved by the Board of Animal Welfare of the National Taiwan University College of Medicine. The stably SPANXA-expressing or mock cells were resuspended at $9 \times 10^{5}$ cells $/ 100 \mu \mathrm{L}$ PBS and injected into the lateral tail vein with a single-cell suspension in PBS. At 5 weeks post-injection, the mice were sacrificed. Their lungs were harvested, fixed in Bouin's solution, and photographed. The numbers of metastatic tumor nodules in the lungs was counted under a dissecting microscope. The representative lung tumors were removed, fixed, and embedded in paraffin. A $5 \mu \mathrm{m}$ section was stained with $\mathrm{H} \& \mathrm{E}$ for histological analysis.

\section{Immunofluorescence}

Stable SPANXA-expressing CL1-5 cells were seeded onto chamber slides $\left(1 \times 10^{3}\right.$ cells/well $)$ before they were incubated for $48 \mathrm{~h}$. After formalin fixing, the cells were blocked and incubated with anti-V 5 at $4^{\circ} \mathrm{C}$ for $16 \mathrm{~h}$. They were then washed using a blocking buffer. Finally, the cells were detected after incubation by using the secondary antibody Alexa Fluor 488 (Life technologies, Carlsbad, CA) for $16 \mathrm{~h}$ at $4^{\circ} \mathrm{C}$. The cell nucleus was counterstained with DAPI, and F-actin was counterstained with Phalloidin (Life technologies, Carlsbad, CA). The cells were imaged on Nikon confocal C1 system.

\section{Statistical analysis}

The statistical tests without annotations were twosided Student $t$ test, and $P$-value $<0.05$ was considered statistically significant. When appropriate, the results are presented as the means \pm SD. SPSS version 13.0 was used for above statistical analyses. Early-stage lung adenocarcinoma cohort (45 patients) used for validation was from GEO database (GSE19188) [44]. We focused the 45 adenocarcinoma lung cancer in the cohort. A patient's risk score was calculated as the sum of the levels of expression of 220922_s_at (SPANXA1/A2, SPANXB1/B2, SPANXC and SPANXF1) and 224032_X at (SPANXA1/A2 and SPANXC). Patients were classified as having a high-risk gene signature or a low-risk gene signature, with the median of risk score as the threshold value. Survival curves for both groups were obtained by the Kaplan-Meier method and were compared using the log-rank test. Both the log-rank test was two-sided, and a $P$-value $<0.05$ was considered statistically significant.

\section{ACKNOWLEDGMENTS}

We thank Dr. Wayne V. Vedeckis of Department of Biochemistry \& Molecular Biology, Louisiana State University Health Sciences Center, Dr. Nan-Haw Chow of Institute of Basic Medical Sciences, National Cheng Kung University, and Dr. Huei-Mei Huang of Graduate Institute of Medical Sciences, Taipei Medical University for providing the construct of pGL3 c-Jun promoter. We also thank the National RNAi Core Facility and the Integrated Core Facility for Functional Genomics of the National Core Facility Program for Biotechnology, the Microarray Core Facility of the National Taiwan University Center of Genomic Medicine for technical support.

\section{CONFLICTS OF INTEREST}

The authors declare that there is no conflicts of interest.

\section{GRANT SUPPORT}

This study was supported by grants from the Ministry of Science and Technology, Taiwan, R.O.C (NSC98-2314B-002-120-MY3, NSC 102-2911-I-002-303, MOST1032911-I-002-303, MOST104-2911-I-002-302) and MIB, 
Institute of Statistical Science, Academia Sinica (Grant No. 5202402010-6). The funders had no role in study design, data collection and analysis, decision to publish, or preparation of the manuscript.

\section{REFERENCES}

1. Siegel R, Ma J, Zou Z, Jemal A. Cancer statistics, 2014. CA Cancer J Clin. 2014; 64:9-29.

2. Chen Z, Fillmore CM, Hammerman PS, Kim CF, Wong KK. Non-small-cell lung cancers: a heterogeneous set of diseases. Nat Rev Cancer. 2014; 14:535-546.

3. Hanahan D, Weinberg RA. Hallmarks of cancer: the next generation. Cell. 2011; 144:646-674.

4. Hajra KM, Chen DY, Fearon ER. The SLUG zinc-finger protein represses E-cadherin in breast cancer. Cancer Res. 2002; 62:1613-1618.

5. Puisieux A, Brabletz T, Caramel J. Oncogenic roles of EMT-inducing transcription factors. Nat Cell Biol. 2014; $16: 488-494$.

6. Gardi NL, Deshpande TU, Kamble SC, Budhe SR, Bapat SA. Discrete molecular classes of ovarian cancer suggestive of unique mechanisms of transformation and metastases. Clin Cancer Res. 2014; 20:87-99.

7. Shih JY, Tsai MF, Chang TH, Chang YL, Yuan A, Yu CJ, Lin SB, Liou GY, Lee ML, Chen JJ, Hong TM, Yang SC, $\mathrm{Su}$ JL, Lee YC, Yang PC. Transcription repressor slug promotes carcinoma invasion and predicts outcome of patients with lung adenocarcinoma. Clin Cancer Res. 2005; 11:8070-8078.

8. Martinez-Estrada OM, Culleres A, Soriano FX, Peinado H, Bolos V, Martinez FO, Reina M, Cano A, Fabre M, Vilaro S. The transcription factors Slug and Snail act as repressors of Claudin-1 expression in epithelial cells. Biochem J. 2006; 394:449-457.

9. Chen JJ, Peck K, Hong TM, Yang SC, Sher YP, Shih JY, Wu R, Cheng JL, Roffler SR, Wu CW, Yang PC. Global analysis of gene expression in invasion by a lung cancer model. Cancer Res. 2001; 61:5223-5230.

10. Wang CC, Tsai MF, Hong TM, Chang GC, Chen CY, Yang WM, Chen JJ, Yang PC. The transcriptional factor YY1 upregulates the novel invasion suppressor HLJ1 expression and inhibits cancer cell invasion. Oncogene. 2005; 24:4081-4093.

11. Chen $\mathrm{CH}$, Chuang SM, Yang MF, Liao JW, Yu SL, Chen JJ. A novel function of YWHAZ/beta-catenin axis in promoting epithelial-mesenchymal transition and lung cancer metastasis. Mol Cancer Res. 2012; 10:1319-1331.

12. Chen CC, Chen HY, Su KY, Hong QS, Yan BS, Chen CH, Pan SH, Chang YL, Wang CJ, Hung PF, Yuan S, Chang GC, Chen JJ, et al. Shisa3 is associated with prolonged survival through promoting beta-catenin degradation in lung cancer. Am J Respir Crit Care Med. 2014; 190:433-444.
13. Semi K, Matsuda Y, Ohnishi K, Yamada Y. Cellular reprogramming and cancer development. Int J Cancer. $2013 ; 132: 1240-1248$.

14. Zendman AJ, Cornelissen IM, Weidle UH, Ruiter DJ, van Muijen GN. CTp11, a novel member of the family of human cancer/testis antigens. Cancer Res. 1999; 59: 6223-6229.

15. Zendman AJ, Zschocke J, van Kraats AA, de Wit NJ, Kurpisz M, Weidle UH, Ruiter DJ, Weiss EH, van Muijen GN. The human SPANX multigene family: genomic organization, alignment and expression in male germ cells and tumor cell lines. Gene. 2003; 309:125-133.

16. Westbrook VA, Diekman AB, Klotz KL, Khole VV, von Kap-Herr C, Golden WL, Eddy RL, Shows TB, Stoler MH, Lee CY, Flickinger CJ, Herr JC. Spermatidspecific expression of the novel $\mathrm{X}$-linked gene product SPAN-X localized to the nucleus of human spermatozoa. Biol Reprod. 2000; 63:469-481.

17. Westbrook VA, Schoppee PD, Diekman AB, Klotz KL, Allietta M, Hogan KT, Slingluff CL, Patterson JW, Frierson HF, Irvin WP, Jr., Flickinger CJ, Coppola MA, Herr JC. Genomic organization, incidence, and localization of the SPAN-x family of cancer-testis antigens in melanoma tumors and cell lines. Clin Cancer Res. 2004; 10:101-112.

18. Salemi M, Calogero AE, Di Benedetto D, Cosentino A, Barone N, Rappazzo G, Vicari E. Expression of SPANX proteins in human-ejaculated spermatozoa and sperm precursors. Int J Androl. 2004; 27:134-139.

19. Westbrook VA, Schoppee PD, Vanage GR, Klotz KL, Diekman AB, Flickinger CJ, Coppola MA, Herr JC. Hominoid-specific SPANXA/D genes demonstrate differential expression in individuals and protein localization to a distinct nuclear envelope domain during spermatid morphogenesis. Mol Hum Reprod. 2006; 12: 703-716.

20. Wang Z, Zhang Y, Liu H, Salati E, Chiriva-Internati M, Lim SH. Gene expression and immunologic consequence of $\mathrm{SPAN}-\mathrm{Xb}$ in myeloma and other hematologic malignancies. Blood. 2003; 101:955-960.

21. Zamuner FT, Karia BT, de Oliveira CZ, Santos CR, Carvalho AL, Vettore AL. A Comprehensive Expression Analysis of Cancer Testis Antigens in Head and Neck Squamous Cell Carcinoma Revels MAGEA3/6 as a Marker for Recurrence. Mol Cancer Ther. 2015; 14:828-834.

22. Yilmaz-Ozcan S, Sade A, Kucukkaraduman B, Kaygusuz Y, Senses KM, Banerjee S, Gure AO. Epigenetic mechanisms underlying the dynamic expression of cancer-testis genes, PAGE2, -2B and SPANX-B, during mesenchymal-toepithelial transition. PloS one. 2014; 9:e107905.

23. Salemi M, Calogero AE, Zaccarello G, Castiglione R, Cosentino A, Campagna C, Vicari E, Rappazzo G. Expression of SPANX proteins in normal prostatic tissue and in prostate cancer. Eur J Histochem. 2010; 54:e41. 
24. Kouprina N, Pavlicek A, Noskov VN, Solomon G, Otstot J, Isaacs W, Carpten JD, Trent JM, Schleutker J, Barrett JC, Jurka J, Larionov V. Dynamic structure of the SPANX gene cluster mapped to the prostate cancer susceptibility locus HPCX at Xq27. Genome Res. 2005; 15:1477-1486.

25. Sakai D, Tanaka Y, Endo Y, Osumi N, Okamoto H, Wakamatsu Y. Regulation of Slug transcription in embryonic ectoderm by beta-catenin-Lef/Tcf and BMPSmad signaling. Dev Growth Differ. 2005; 47:471-482.

26. Saegusa M, Hashimura M, Kuwata T, Okayasu I. Requirement of the Akt/beta-catenin pathway for uterine carcinosarcoma genesis, modulating E-cadherin expression through the transactivation of slug. Am J Pathol. 2009; 174:2107-2115.

27. Tajeddine N, Gala JL, Louis M, Van Schoor M, Tombal B, Gailly P. Tumor-associated antigen preferentially expressed antigen of melanoma (PRAME) induces caspaseindependent cell death in vitro and reduces tumorigenicity in vivo. Cancer Res. 2005; 65:7348-7355.

28. Kalashnikova EV, Revenko AS, Gemo AT, Andrews NP, Tepper CG, Zou JX, Cardiff RD, Borowsky AD, Chen HW. ANCCA/ATAD2 overexpression identifies breast cancer patients with poor prognosis, acting to drive proliferation and survival of triple-negative cells through control of B-Myb and EZH2. Cancer Res. 2010; 70:9402-9412.

29. Iles RK. Ectopic hCGbeta expression by epithelial cancer: malignant behaviour, metastasis and inhibition of tumor cell apoptosis. Mol Cell Endocrinol. 2007; 260-262:264-270.

30. Song $\mathrm{MH}$, Choi KU, Shin $\mathrm{DH}$, Lee $\mathrm{CH}$, Lee SY. Identification of the cancer/testis antigens AKAP3 and CTp11 by SEREX in hepatocellular carcinoma. Oncol Rep. 2012; 28:1792-1798.

31. Chen Z, Li M, Yuan Y, Wang Q, Yan L, Gu J. Cancer/testis antigens and clinical risk factors for liver metastasis of colorectal cancer: a predictive panel. Dis Colon Rectum. 2010; 53:31-38.

32. Yang P, Huo Z, Liao H, Zhou Q. Cancer/testis antigens trigger epithelial-mesenchymal transition and genesis of cancer stem-like cells. Curr Pharm Des. 2015; 21: 1292-1300.

33. Eferl R, Wagner EF. AP-1: a double-edged sword in tumorigenesis. Nat Rev Cancer. 2003; 3:859-868.

34. Kappelmann M, Bosserhoff A, Kuphal S. AP-1/c-Jun transcription factors: regulation and function in malignant melanoma. Eur J Cell Biol. 2014; 93:76-81.
35. Yan L, Della Coletta L, Powell KL, Shen J, Thames H, Aldaz CM, MacLeod MC. Activation of the canonical Wnt/ beta-catenin pathway in ATF3-induced mammary tumors. PloS one. 2011; 6:e16515.

36. Nguyen PT, Tsunematsu T, Yanagisawa S, Kudo Y, Miyauchi M, Kamata N, Takata T. The FGFR1 inhibitor PD173074 induces mesenchymal-epithelial transition through the transcription factor AP-1. Br J Cancer. 2013; 109:2248-2258.

37. Chen H, Zhu G, Li Y, Padia RN, Dong Z, Pan ZK, Liu K, Huang S. Extracellular signal-regulated kinase signaling pathway regulates breast cancer cell migration by maintaining slug expression. Cancer Res. 2009; 69: 9228-9235.

38. Ramsdale R, Jorissen RN, Li FZ, Al-Obaidi S, Ward T, Sheppard KE, Bukczynska PE, Young RJ, Boyle SE, Shackleton M, Bollag G, Long GV, Tulchinsky E, et al. The transcription cofactor c-JUN mediates phenotype switching and BRAF inhibitor resistance in melanoma. Sci Signal. 2015; 8:ra82.

39. Liu J, Han Q, Peng T, Peng M, Wei B, Li D, Wang X, Yu S, Yang J, Cao S, Huang K, Hutchins AP, Liu H, et al. The oncogene c-Jun impedes somatic cell reprogramming. Nat Cell Biol. 2015; 17:856-867.

40. Gerald D, Chintharlapalli S, Augustin HG, Benjamin LE. Angiopoietin-2: an attractive target for improved antiangiogenic tumor therapy. Cancer Res. 2013; 73: $1649-1657$.

41. Imanishi Y, Hu B, Jarzynka MJ, Guo P, Elishaev E, Bar-Joseph I, Cheng SY. Angiopoietin-2 stimulates breast cancer metastasis through the alpha(5)beta(1) integrinmediated pathway. Cancer Res. 2007; 67:4254-4263.

42. Dong YD, Cui L, Peng CH, Cheng DF, Han BS, Huang F. Expression and clinical significance of HMGB1 in human liver cancer: Knockdown inhibits tumor growth and metastasis in vitro and in vivo. Oncol Rep. 2013; 29:87-94.

43. Chang YH, Chen CM, Chen HY, Yang PC. Pathwaybased gene signatures predicting clinical outcome of lung adenocarcinoma. Sci Rep. 2015; 5:10979.

44. Hou J, Aerts J, den Hamer B, van Ijcken W, den Bakker M, Riegman P, van der Leest C, van der Spek P, Foekens JA, Hoogsteden HC, Grosveld F, Philipsen S. Gene expressionbased classification of non-small cell lung carcinomas and survival prediction. PloS one. 2010; 5:e10312. 